\title{
Development of the grain energy bars with the high content of dietary fibers
}

\author{
Irina Krasina ${ }^{1, *}$, Anna Kurakina ${ }^{1}$, Cholpon Kasymova ${ }^{2}$, and Ekaterina Krasina ${ }^{1}$ \\ ${ }^{1}$ Kuban State Technological University, Russia \\ ${ }^{2}$ Kyrgyz State Technical University named after Razzakov, Kyrgyzstan
}

\begin{abstract}
The research results on the development of grain energy bars are given. The experimental plan for the mixtures was used to create the grain energy bars with prebiotic properties. Inulin, oligofructose and gum-arabic were selected as prebiotic ingredients, which were added to the binding syrup, and subsequently to cereals and fruits. Grain energy bars were analyzed by quantitative descriptive analysis and the best formulae were determined according to the preference test. It was found that the gum-arabic was responsible for the influence on the appearance of cereal flake dryness, hardness and chewability, while the oligofructosis increased the color brightness and the crunch when bitten. The optimized composition of the grain energy bar sample showed that the fiber mixture gave the bars better texture characteristics than each fiber individually. The viscosity of the syrup, which is strongly influenced by the gum-arabic concentration, had a negative correlation with the consumer preference assessment. The selected recipe of the grain energy bar allows to reduce energy value by $18-20 \%$ with simultaneous increasing of total amount of dietary fibers by $200 \%$.
\end{abstract}

The demand for quality and safe food is growing all over the world, and the consumption of balanced food is the right way to avoid or correct the health problems such as obesity, diabetes, cardiovascular diseases, which are largely determined by dietary errors and are alimentary dependent [1]. In products intended for sports nutrition, the list of ingredients should contain a sufficient amount of proteins and carbohydrates, with a small amount of fat. Today, a large number of foods enriched with vitamins and other useful substances are being developed. When choosing such products, consumers pay attention to the list of ingredients, the quality and the products safety, as well as to their expiry date [2, 3, 4, 5]. In the today's rhythm, athletes need healthy food for a snack between the main meals. Therefore, the creation of such products becomes a high priority. Such products include raw bars that can provide the necessary energy. Grain food bars are made of extruded or whole wheat pulp with sweet and pleasant taste and they are a source of vitamins, minerals, dietary fibers, proteins and complex carbohydrates and correspond to this trend [6]. Grain raw bars are multicomponent and could have a very complex formula. The grain raw bar formula ingredients need to be combined appropriately to complement each other in terms of the sensory evaluation, texture and physical properties, especially at the equilibrium point of water activity on which the bar retention depends. Bars made from grains include granola

* Corresponding author: krasina@kubstu.ru 
bars and cereal bars. Grain bars are a mixture of cereals with other products, such as nuts and fruits, forming a bar of this mixture with binding components. Now the search is under way for new ingredients that could meet the consumer market requirements. The consumer market is going to get products with the high sensory and nutritional qualities bringing benefits to the health [7]. In the development of new products, the optimization of parameters such as shape, color, appearance, taste, texture and consistency is very important, as well as the interaction of various components to achieve a complete balance that leads to a high quality final product demanded by consumers. Traditionally, ingredients containing insoluble dietary fibers such as bran, for example, have been used in food products such as grain raw bars. But brans palatability has limited the level that can be incorporated into various food systems. At the present day, the ingredients containing soluble fiber arouse interest in the development of "healthy" products because they are more pleasant to the taste. In addition, in the food systems some of them can be used for densifying, increasing viscosity or gelling [8].

As far as is known [8.9], the dietary fibers contribute to productive physiological effects, including the laxative effect, blood cholesterol level lowering, and/or blood glucose decreasing. In addition to the laxative action, perhaps the most dynamic function of the dietary fibers is the fermentation, which causes many biochemical, physiological and microbiological changes in the large intestine.

Inulin, oligofructose and gum-arabic are well suited soluble fibers for using in diabetic or low- calorie food products. These ingredients also have a variety of technological functional properties: water retention, viscosity increasing for binding and texture improving, stability at various temperature levels and stable $\mathrm{pH}[10]$.

The aim of our study was to investigate the effect of the quantitative dietary fiber content in grain energy bars on their properties, nutritional value, and the consumer demand for texture and sensory characteristics optimization. Inulin, oligofructose and gum-arabic have been used as sources of dietary fibers in the development of the grain energy bars.

A simplex centroid design was used for mixtures of three components with two centroids rerun points to study the interaction effects between ingredients on the product sensory quality. The process of preparing the grain energy bars mass was carried out in 2 stages: first, a solution of binding syrup was prepared, and then it was added to a mixture of dry ingredients consisting of grain components, nuts and dried fruits, and thoroughly mixed. The ratio of syrup to dry components was 30:70. The finished mass was formed into a $2.5 \mathrm{~cm}$ thick formation and cut into bars. The tepid bars were analyzed according to physicochemical and organoleptic indicators, as well as texture. The mathematical processing of the experimental data was carried out using Statistica software for Windows 7.0.

During the experimental research, 9 development prototypes were prepared with a different ratio of components in the mixture from 0 to $100 \%$. The sensory evaluation of the samples was carried out on a nine-point hedonistic scale, a result of which presented in the Table 1. Chewiness was defined as the amount of mouth-feel (one mouth-feel/ per sec) needed for reducing the 2 cc of the sample for an optimum consistency for dilution.

Table 1. Average scores for each examined attribute and chewable capacity.

\begin{tabular}{|l|c|c|c|c|c|c|c|c|}
\hline Sample & Colour & Dryness & Aroma & Sweetness & Taste & Hardness & Crunch & Chewability \\
\hline 1 & 3,61 & 4,08 & 3,01 & 3,36 & 4,07 & 3,53 & 3,24 & 29,33 \\
\hline 2 & 5,63 & 1,53 & 2,88 & 4,82 & 3,85 & 2,07 & 6,48 & 24,25 \\
\hline 3 & 2,33 & 6,32 & 4,78 & 3,23 & 3,93 & 5,04 & 2,56 & 34,08 \\
\hline 4 & 4,73 & 2,72 & 2,13 & 4,26 & 3,60 & 2,74 & 4,07 & 25,50 \\
\hline 5 & 2,25 & 6,24 & 3,43 & 3,45 & 3,94 & 4,42 & 3,39 & 28,42 \\
\hline 6 & 3,12 & 5,49 & 4,56 & 3,54 & 3,48 & 3,33 & 4,58 & 29,08 \\
\hline 7 & 3,47 & 3,72 & 3,18 & 3,78 & 4,24 & 2,72 & 3,53 & 27,75 \\
\hline 8 & 3,28 & 5,25 & 3,30 & 3,79 & 3,23 & 2,89 & 4,36 & 27,58 \\
\hline 9 & 4,13 & 3,96 & 3,47 & 4,08 & 3,31 & 3,59 & 3,67 & 27,50 \\
\hline
\end{tabular}


As can be seen from the data presented, the taste of all grain energy bars samples is estimated at approximately the same level. The level of oligofructose in the bar formula has the greatest impact on such characteristics as color, sweetness and crunch, while the gumarabic has the least effect on these indicators. Oligofructose also improves the external perception of the grain energy bar, while the addition of gum-arabic leads to the transparency decreasing, as well as increases the grain mixture dryness. Gum-arabic contributed to the enhancement of the bar fruit smell, and the values decreasing of this indicator in samples, with a mixture of inulin and oligofructose, as well as inulin and gum- arabic, pointed at antagonistic effects during their interaction. The increase of the sweetness values while introducing inulin and oligofructose is due to the fact that they have a slightly sweet flavour without aftertaste. Oligofructose gives a sweet taste profile similar to sucrose taste profile while combining with other sweeteners, but only up to $30 \%$ sweetness. But in the syrup composition the neutral flavored gum-arabic enhances the sweetness of isomalt and molasses.

Gum-arabic and inulin have an effect on the hardness increasing of grain energy bars, while the oligofructose usage gives the opposite effect and contributes to the hardness reducing. The samples with a higher degree of embrittlement were obtained during increasing of the oligofructose concentration, while the gum-arabic addition to the fiber mixture contributed to a stronger decrease of this trait. A grain energy bar with only gum-arabic usage has the highest score of the chewability, while a bar with oligofructose has the lowest score. Most consumers note that the crunchy feel is desirable in a grain energy bar, but chewing texture is much preferred. Thus, it can be noted that the differences in textures and appearance of the grain energy bars are greater than the differences between flavors and tastes, because the added dietary fibers influenced the texture and appearance of the grain energy bars more than their aroma and taste.

Inulin and oligofructose had a stronger and more similar effect on consumer choice, as demonstrated by higher sensory scores. The binary systems of inulin-oligofructose and oligofructose-gum-arabic showed a synergistic effect, while the mixture of inulin-gum-arabic showed an antagonistic effect. The samples with a higher sensory score ( $>7$ points) were obtained in an experimental zone with a higher proportion of oligofructose in the fiber mixture. Considering the texture importance in the proposed formulas of grain energy bars, while optimizing the combined texture characteristics - minimum hardness, maximum chewability and maximum crispness - the following prebiotic fibers mixture was chosen: 8.46\% inulin, $66.16 \%$ oligofructose, $25.38 \%$ gum arabic.

The viscosity of the syrup can affect some sensory characteristics of grain energy bars. The syrup containing 100\% gum-arabic had a higher viscosity - 213 times higher than the syrup with $100 \%$ oligofructose and 103 times higher the viscosity of the syrup with $100 \%$ inulin (Fig. 1).

The higher viscosity of the syrup containing $100 \%$ gum-arabic is due to its molecular weight, which is about 200 times higher than that of oligofructose. The gum-arabic is considered as a complex, highly branched, globular molecule that is not linear and densely packed, which explains its viscosity. The syrup viscosity of each sample showed the negative correlation with the sensory preferences of grain energy bars $(r=-0.904, p=0.0008)$ and the positive correlation with the hardness of grain energy bars $(r=0.833, p=0.0053)$. In addition, the syrup viscosity was positively correlated with the determined instrumentally hardness ( $\mathrm{r}$ $=0.792, p=0.0388$ ). In this way, if the syrup viscosity is higher, the grain energy bar will be harder, which is undesirable for the consumer. In addition, the fiber mixture has proved to be very effective in terms of technological functional properties in terms of its influence the sensory characteristics of the bars. Inulin and gum-arabic helped to control grain mass flowability at low temperatures, which facilitates the bar shape retention and simplifies its packaging. The grain energy bar prepared with $100 \%$ oligofructose was sticky and very soft, 
the bar with $100 \%$ inulin was solid, and the bar, containing $100 \%$ of gum-arabic, was solid and dry (Table 1). Oligofructose can be used as a part of the binding system as a humectant to make the bar softer and more pliable throughout its shelf life, and inulin acts as a texture modifier, keeping moisture, which helps keep the bars fresh for longer.

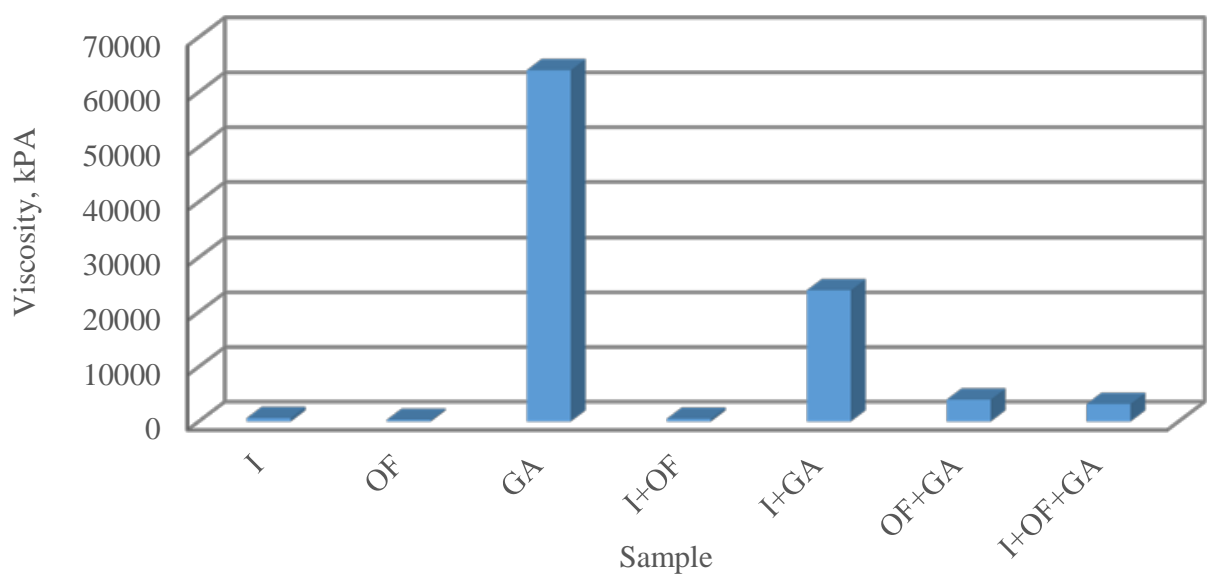

Fig. 1. The viscosity of syrup at $80^{\circ} \mathrm{C}$, containing inulin (I), oligofructose (OF) and gum arabic (GA).

The determination of the chemical composition of the grain energy bar (Table 2) sample prepared according to the optimized formula showed a calorie reduction by $18-20 \%$, while providing the dietary fiber content increasing by $200 \%$, in comparison with the control sample, prepared without inulin, oligofructose and gum-arabic.

Table 2. The nutrition value of grain energy bars.

\begin{tabular}{|c|c|c|}
\hline Indicators & \multicolumn{2}{|c|}{ Content, g/100g } \\
\hline & control & control \\
\hline Moisture & 5,47 & 6,16 \\
\hline Protein & 6,11 & 5,58 \\
\hline Fat & 4,13 & 2,60 \\
\hline Carbohydrates & 75,70 & 61,40 \\
\hline Dietary fibers & 7,08 & 22,65 \\
\hline Ash & 1,51 & 1,61 \\
\hline Energy value, kcal & 364,36 & 291,24 \\
\hline
\end{tabular}

Thus, based on the research conducted, it can be concluded that the mixture of inulin dietary fibers, oligofructose and gum-arabic has the necessary technologically functional properties and can be used to improve the sensory characteristics of grain energy bars. The addition of inulin, oligofructose and gum-arabic to the grain energy bar allows to reduce energy value by $18-20 \%$ while providing $200 \%$ of added dietary fibers. Using the possibilities of the mathematical development of experimental mixtures and organoleptic examination, it became possible to optimize the formula of a nutritious, tasty, as well as ready-to-eat product with the declared functional properties as a source of prebiotic fibers. Thus, this grain energy bar has a good opportunity for the market launch in order to provide the health benefits to both athletes and the population as a whole. 


\section{References}

1. M.N.Kim The Eurasian Union of Scientists. 1-2(22), 65-68 (2016).

2. I.B.Krasina, N.V.Hodus Successes of modern natural science. 9, 92-93 (2004)

3. F.Vanhonacker, B.Kühne, X.Gellynck, L. Guerrero, M.Hersleth, W. Verbeke Food Res. Int. 54 (2), 1828-1835 (2013)

4. I.Y.Reznichenko, N.Y.Ruban Technology and commodity science of innovative food products. 5 (64), 57-61 2020

5. I.B.Krasina, I.N.Bezuglaya, V.V.Nersesyan Successes of modern natural science 5, 5253 (2005)

6. N.Rawat, I.Darappa, J Food Sci Technol 52, 3006-3013 (2015)

7. L.A.Mayurnikova, A.A.Koksharov, T.V.Krapiva Technic and technology of food production facility. 50(1), 124-139 (2020).

8. T.A.Karachanskaya, I.B.Krasina, N.K.Danovich, A.V.Krasyuk, University proceedings. Product technology 4, 43-44 (2010)

9. E.I.Melnikova, E.S.Skrynikova, E.S.Rudnichenko University proceedings. Product technology 4, 62-63 (2013)

10. E.R.Serbaeva, A.B.Yakupova, Y.R.Magasumova, K.A.Farkhutdinov, G.R.Akhmetova, B.R.Kuluyev Biomics. 12 (1), 57-79 (2020) 